\title{
Methodology for Transformation of Individual Scan Data into Realistic Animated Human Models
}

\author{
Christine MEIXNER* , Sybille KRZYWINSKI ${ }^{2}$ \\ ${ }^{1}$ Laloni GmbH, Nusshof, Switzerland; \\ 2 TU Dresden, Germany
}

http://dx.doi.org/10.15221/14.337

\begin{abstract}
Animating individual scan data in an efficient and easy way is still quite a big challenge. Even more if anatomical correctness is required concerning the deformation by body movement.

We present a methodology for transforming individual scan data into realistic animated human models by adapting the scan data to a template model based on anthropometric coherences (skeleton, muscles, tendons and chondrals). The methodology is demonstrated for a female body. It can be used for a male body or even fictive creatures as well. For modeling and animation $3 d s$ Max is used, but the technique is not fixed to a specific commercial system.

This approach allows an easy examination of the correlation between the posture of a person and the ideal pattern for a specific pose or movement. As the pose of an athlete differs significantly from the standard standing pose this solution can be used for garment design for high-performance sports. Another possible application is the construction and comparison of patterns in standing and sitting position to be used for wheel-chaired people.
\end{abstract}

Keywords: 3d body scanning, Anthropometric studies, Human body modeling, Animation, Simulation, Anatomy, Skeleton, Muscle Systems

\section{Introduction}

Because the polygonal mesh produced by body scanners is unstructured and far too fine (see figure 1) for being animated directly, a representative mesh matching the surface of the scan data is applied. Our methodology adopts the common approach of using template models for time-consuming tasks [1, $4,5]$ and adapting, transferring and interpolating them to individual data. The second part is done widely automatically.
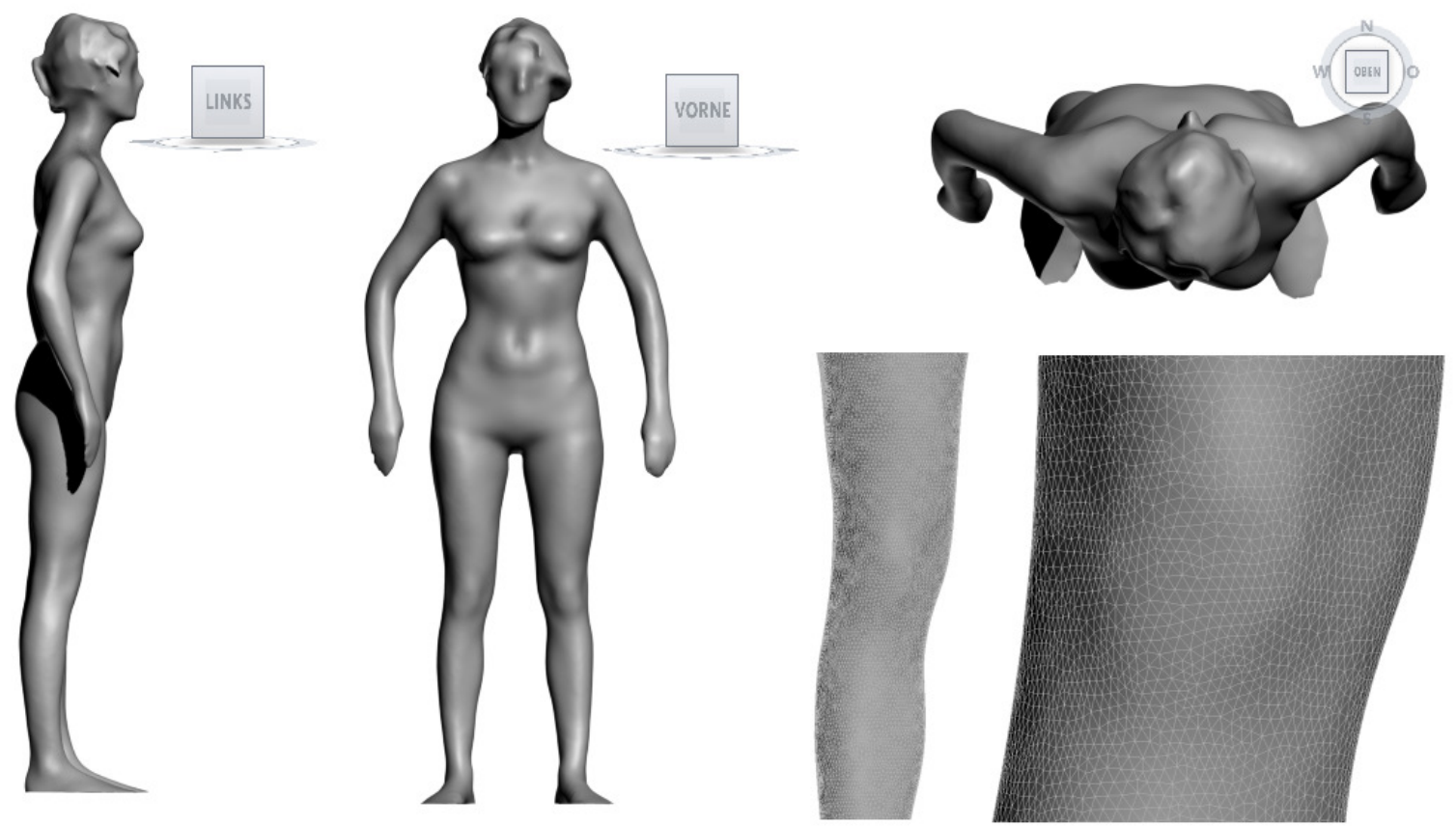

Fig. 1 Sample scan data in left, front and top view and detail of the mesh for a leg / knee. 
First of all, the general procedure is explained for an animated template model composed of a cylinder, two bones and one muscle. As the requirements for the density of the mesh differ for animation and resulting models we examine if the topology of the mesh can be changed in a controlled way during the process. This result has a direct influence on the density of our real template model for the human body. Finally, the surface of the cylinder is assimilated to the scan model and the animation specifications are transferred and interpolated.

To achieve the requirement of reasonable anthropometric movements the template model is based on a three-dimensional MRI (magnetic resonance imaging) model. We use skin, skeleton, muscles, tendons and chondrals of it and call the created animated model an anatomic template. After adapting this to scan data we can animate it in a corresponding way as for the cylinder.

\section{General Approach}

The general approach is demonstrated for a very simple setup consisting of a cylinder, two bones and a muscle.

\subsection{Template Model}

The sample cylinder is defined with 8 sides and 10 cross sections (see Fig. 2a) and assigned to a bones system of two schematic bones (see Fig. 2b). After adjusting the weights the surface of the cylinder follows the movement of the bones already. Fig. 2c shows the weights for the upper bone (red = influence of the selected bone exclusively, yellow = major influence of that bone, blue = only small influence).

After the rigging and skinning process a schematic muscle is attached between the two bones (2d). It follows the movement of the bones system as well and stretches and bulges. The muscle is added to the rig and the skinning is adjusted manually. Fig. $2 e$ shows the resulting model. For preparation the weights table (2f) is written to a file. It contains the vertex numbers and the weight for the two bones and the muscle for each of it. Landmarks (green), joint points (blue) and muscle starting and end point (red) are created and linked to the surface resp. the bones resp. the muscle (see Fig. 2g). All this helper points follow the movement (2h).
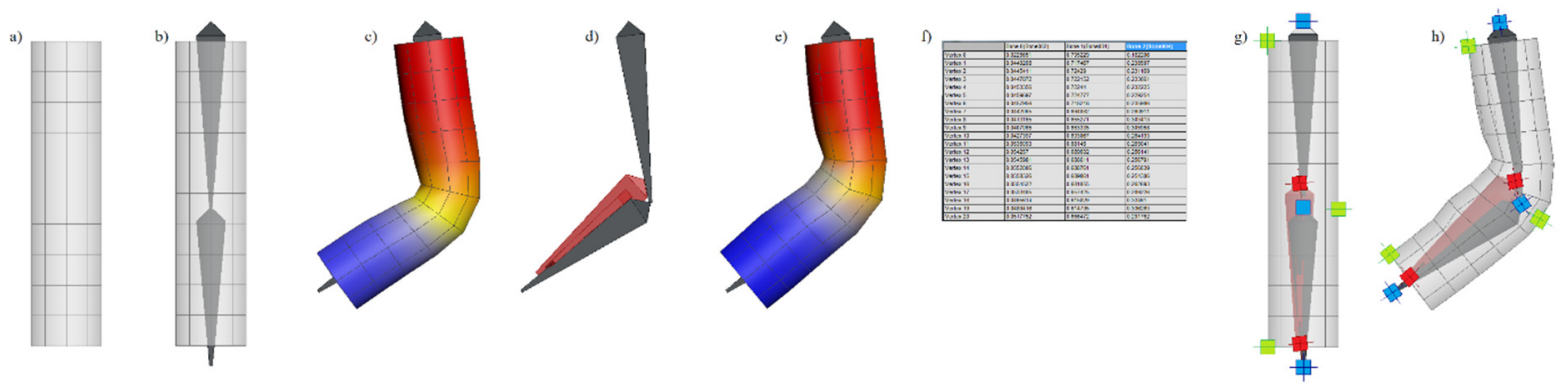

Fig. 2 Creating the template model for a cylinder

\subsection{Changes in Topology of Mesh}

To insert vertices, edges are divided in a defined order (see Fig. 3).
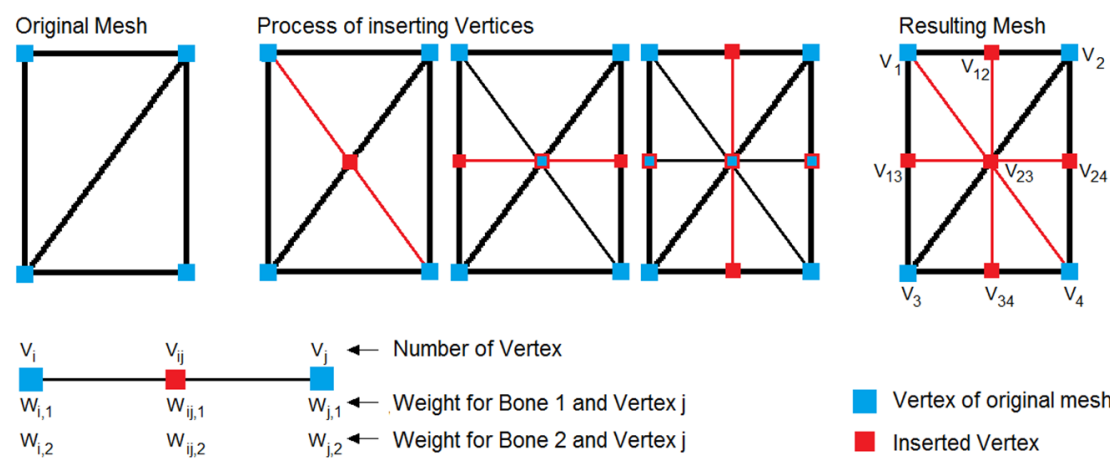

Vertex of original mesh

Inserted Vertex

Fig. 3 Order of inserting vertices to divide a face into 4 equal faces 
Fig. 3 shows the division of each edge into two halves. To keep the general structure of the faces first all diagonals are divided followed by the vertical and horizontal edges. This process is done step by step programmatically. For each new vertex a line containing the new vertex index, the indices of the neighbors and their relative ratio is written to a file.

The weights of the new vertices for bone 1 (equation 1) and bone 2 (equation 2) in the middle of the edge are calculated as follows:

$$
\begin{aligned}
& W_{i j, 1}=0.5 *\left(W_{i, 1}+W_{j, 1}\right) \\
& W_{i j, 2}=0.5 *\left(W_{i, 2}+W_{j, 2}\right)
\end{aligned}
$$

If the ratio of the two new edges is $1: \eta$ the weights are distributed analog to that:

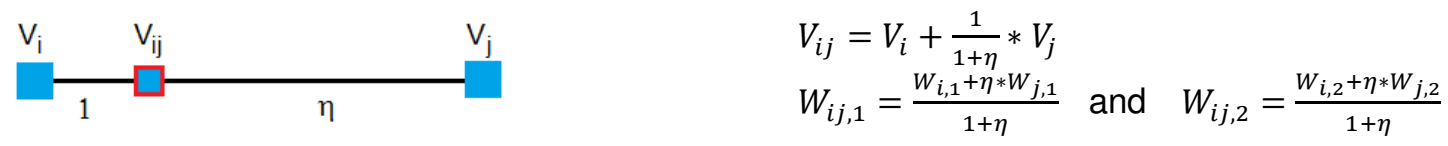

After dividing all edges of the cylinder into two halves, interpolating the weights and setting the weight values for the inserted vertices the surface deforms in a smoother way (see Fig. 4).
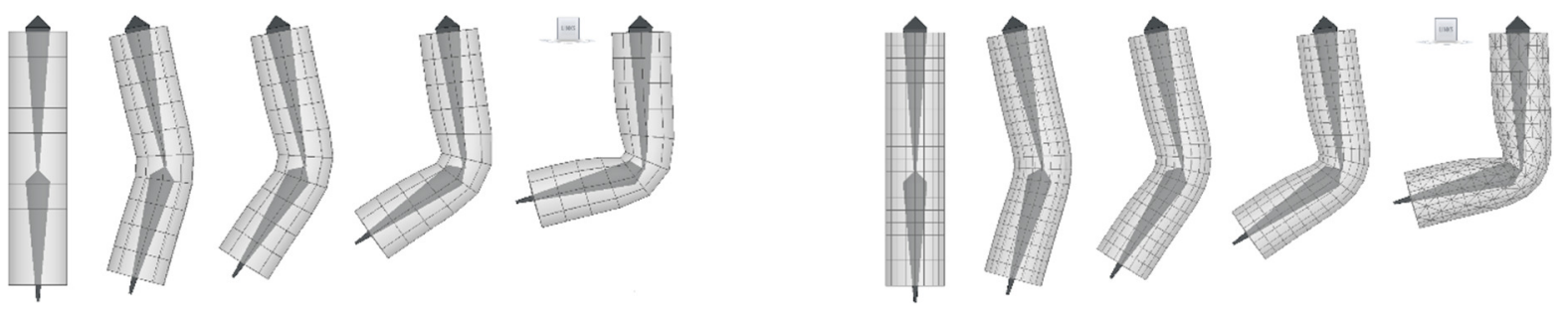

Fig. 4 Deformation of the cylinder before (left) and after (right) refining the mesh

That means that the faces can be divided after the skinning process has been finished and that the weight of the new vertices can be calculated programmatically. Prerequisite therefore is that the definition of the new positions (i.e. indices of neighbor vertices and ratio of each neighbor) is known.

\subsection{Transfer Template to Scan Data}

Now, the template model is adopted to the scanned leg data. First the positions of the joints is guessed and the two bones are scaled (Fig. 7b) and rotated (Fig. 7c) accordingly. Then an intersection plane $E_{S}$ normal to the axis $\vec{b}$ of each bone is determined for each vertex of the cylinder (see Fig. 5 and 6 ).
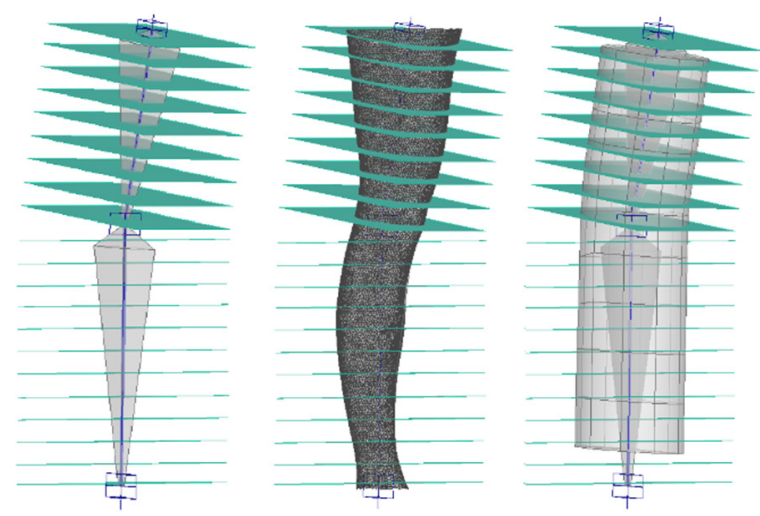

Fig.5 Intersection planes

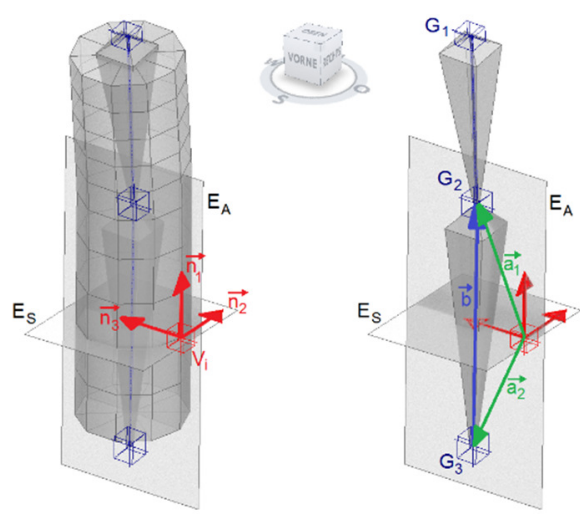

Fig. 6 Intersection plane and normal vectors 
Each of the vertices is moved in the intersection plane towards the bone axis and into the opposite direction. The nearest intersection point is calculated and set as the new value of the vertex. Fig. 7d shows the result of this automated adaption process to the scan data in side and top view.

The faces are marked to divide their edges into two (Fig. 7e, left), three (Fig. 7e, middle) resp. four (Fig. $7 \mathrm{e}$, right) parts, which is done programmatically and leads to the mesh in Fig. 7f. The new inserted vertices are moved to the surface of the leg again by applying the same algorithm as shown in Fig. 6 . Each vertex of the mesh in Fig. $7 \mathrm{~g}$ lies on the surface of the leg data. All scripts write out how the new inserted vertices are defined. This is used now to interpolate the weights for this new points.

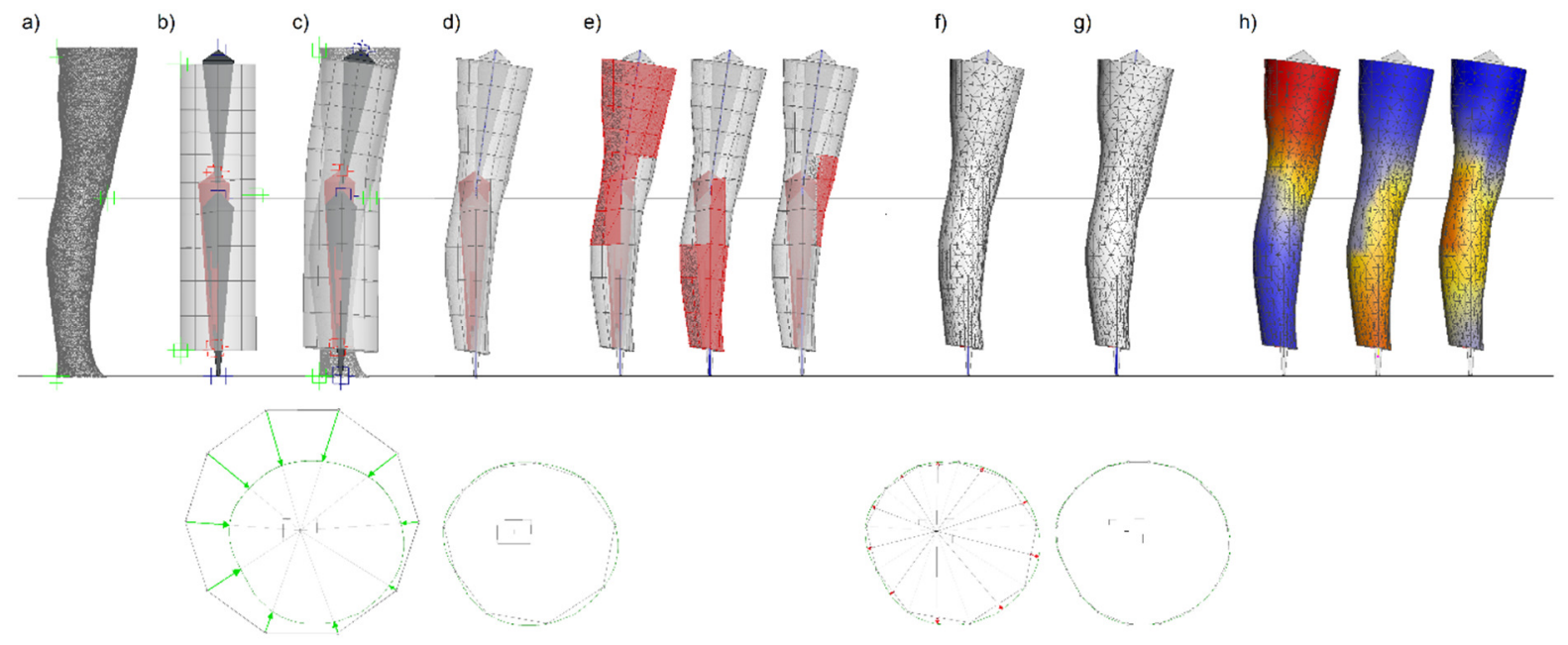

Fig. 7 Process of adapting the template model to the leg data

After transferring all weights (see Fig. 7.h) the leg behaves in exactly the same way as the cylinder did before (see Fig. 8).

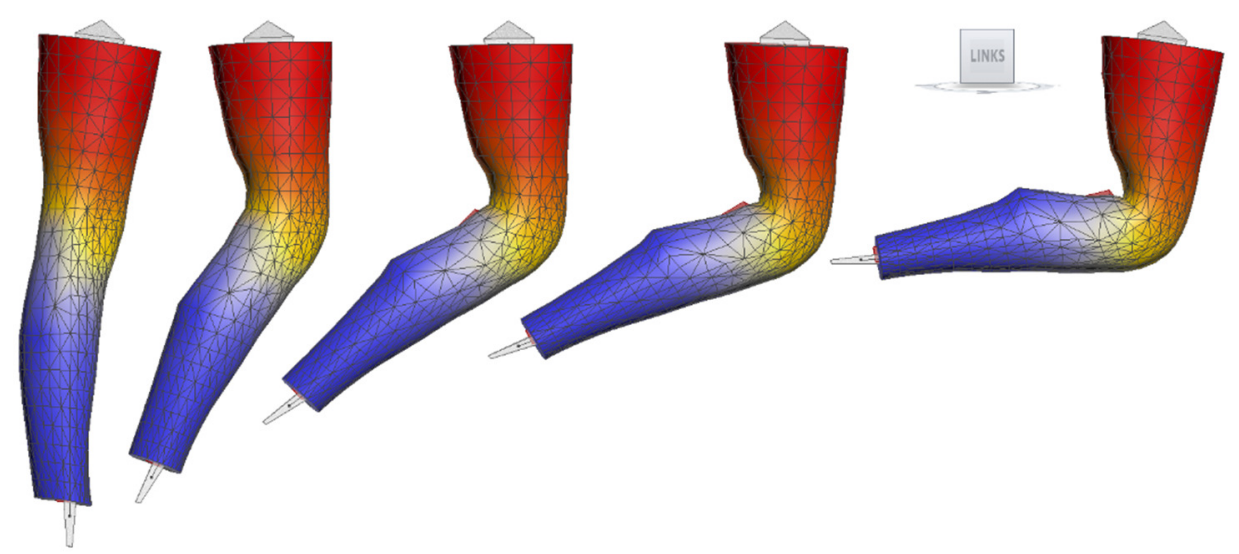

Fig. 8 Deformation of the leg after transferring and interpolating all data from the cylinder

Compared to the deformation of the cylinder (see Fig. 2.h) the leg shows the same characteristics: An sharp corner in the middle of bone 2 (lower leg) and a notch on the backside of the knee. As the leg gets smaller from top to down the effect is a little bit more distinctive than for the cylinder. The transfer process is working in the correct way. But for a lifelike deformation more muscles close to reality are required to be modeled and considered. 


\section{Anatomic Template}

To create a template model with an integrated realistic skeleton and muscle system and reasonable anatomic coherences we used the model Ella, a 26-Year-Old Woman, of the Virtual Population [5] provided by ITIS in cooperation with ETH Zurich $(\mathrm{CH})$ and the University of Erlangen-Nürnberg (D).

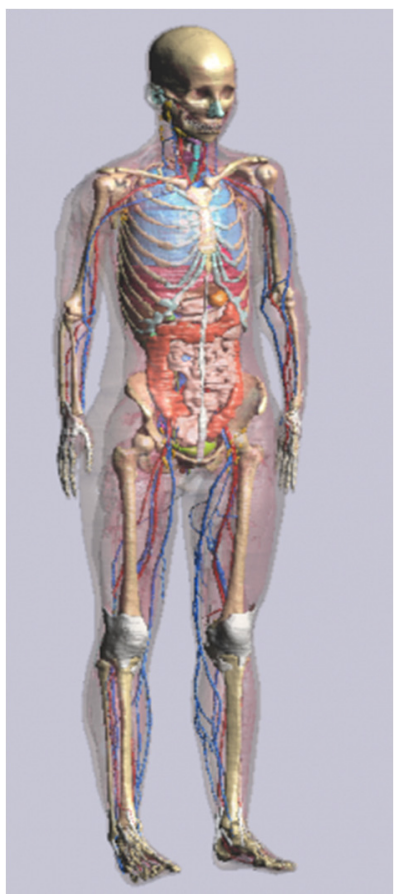

The data were recorded in a MRI-scanner, the model is static. The different layers (skeleton, muscles, skin, fat, blood vessels, organs, etc.) can be switched on and off.

The specifications of the person scanned:

\begin{tabular}{|l|l|}
\hline Sex & Female \\
\hline Type & Young Adult \\
\hline Age $[$ Years] & 26 \\
\hline Height $[\mathrm{m}]$ & 1.63 \\
\hline Weight $[\mathrm{kg}]$ & 58.7 \\
\hline BMI $\left[\mathrm{kg} / \mathrm{m}^{2}\right]$ & 22.0 \\
\hline Number of Tissues & 305 \\
\hline
\end{tabular}

The model cannot be imported directly into 3ds Max or another CAD system, so we took screenshots from different views and used them as background images to create a surface model from scratch.

Only the right half was created and mirrored (see Fig 10).

Fig. 9 Model Ella of the Virtual Population [5]
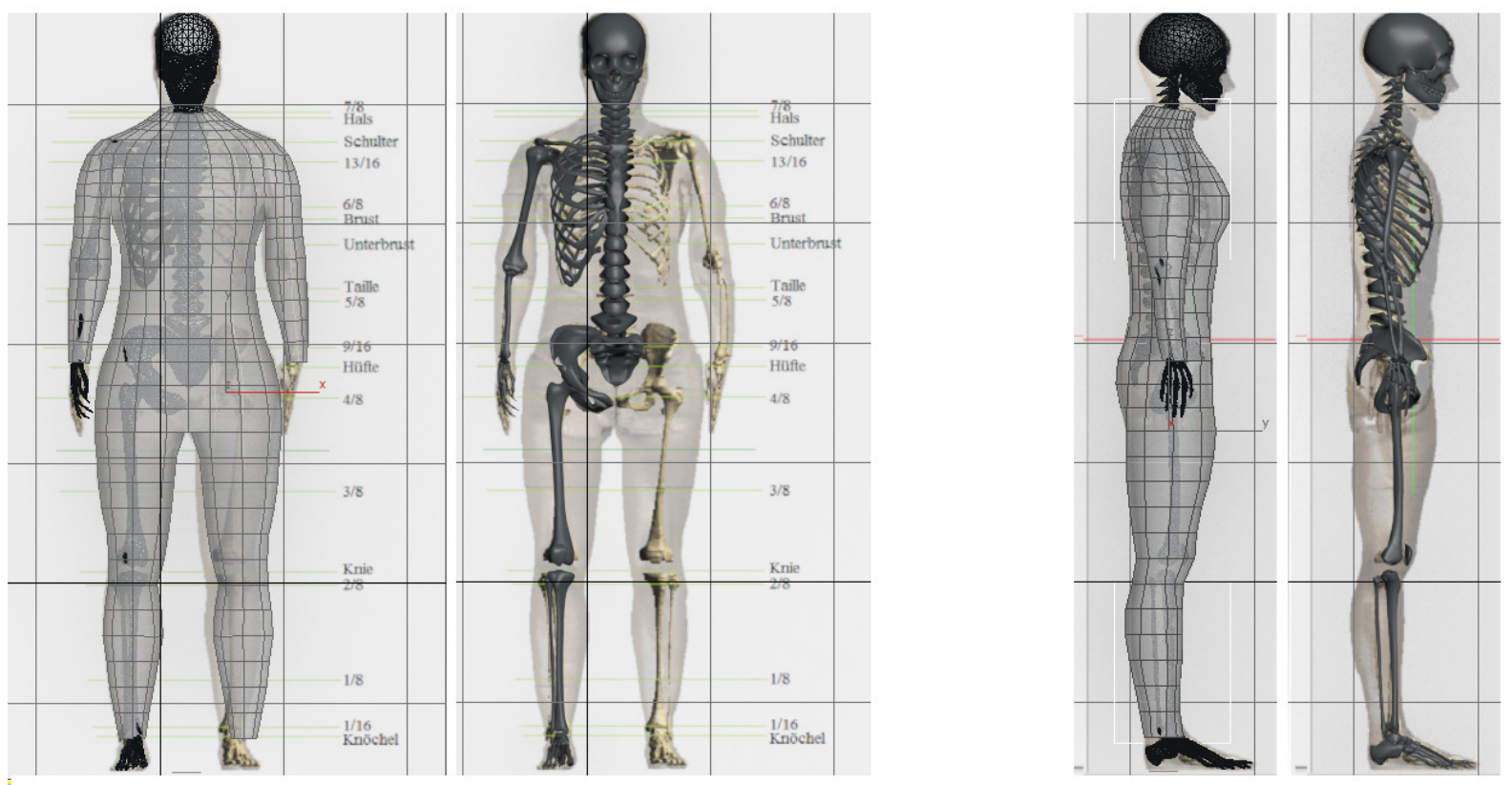

Fig. 10 Background Image of Ella with the newly-created polygonal mesh (in each view left part) and the newly-created right part of the skeleton as 3ds Max model 
The anatomic skeleton is extremely helpful to check if the joints are on the right position and if they stay in that position when moving bones. Fig. 11 (middle) shows that the thigh bone leaves the joint socket at movement. The joint position is wrong in that case. After correcting it the bone rotates around the supposed point and stays in the joint socket (Fig. 11 right).
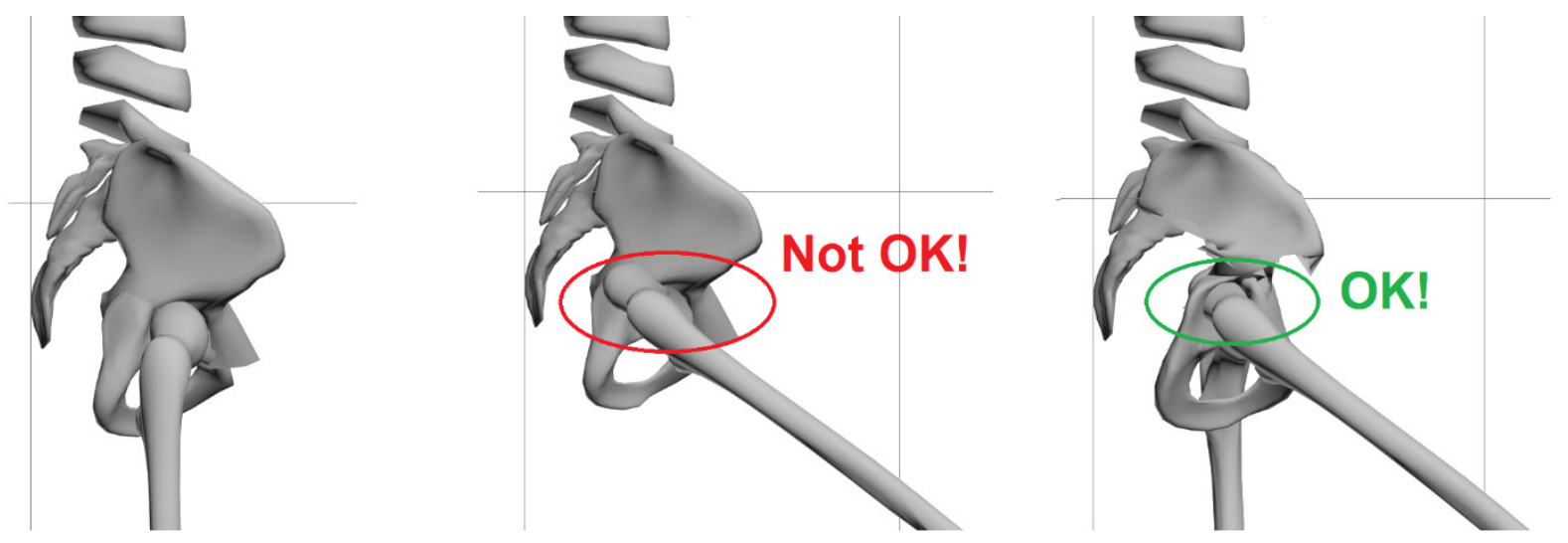

Fig. 11 Each joint has to be controlled carefully if it moves in the correct way

After checking all joints and bone movements the newly-created template surface can be rigged and skinned for the skeleton to map the basic correlations between bones and skin already. As the muscles are missing some artefacts appear at the joints. Deformations caused by muscles cannot be considered as they are not modeled yet.

The rigging process is followed by the creation of the main muscles based on ELLA and anatomic correlations like the movement of the patella and muscles at bending the knee (see image 12)
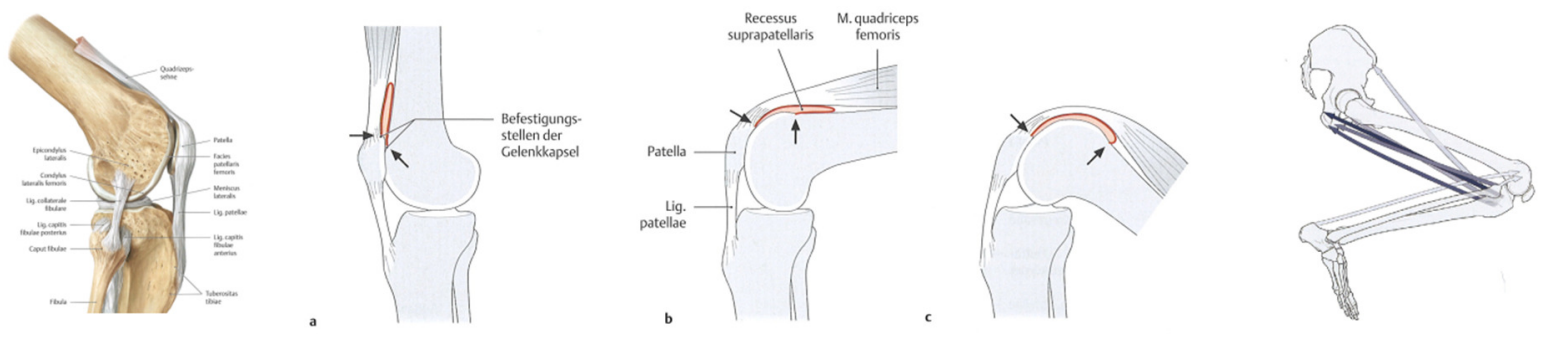

Fig. 12: Anatomic correlations at bending the knee (images are taken from [1])

Three different kind of musculoskeletal parts are modeled:

- postural muscles (e.g. abdominal muscles)

- $\quad$ skeletal muscles (e.g. biceps)

- tendons and chondrals (e.g. patella and its attachment straps) 
These different kind of muscles lead to different skin deformations. The effect of postural muscles is wide, smooth and flat. Skeletal muscles bulge and squeeze a lot and are restricted to a smaller area. Tendons are very concentrated on a tiny area and have the highest influence of all types. Each muscle type has its own structure and behavior. The influence of each muscle on the skin can be exported individually.
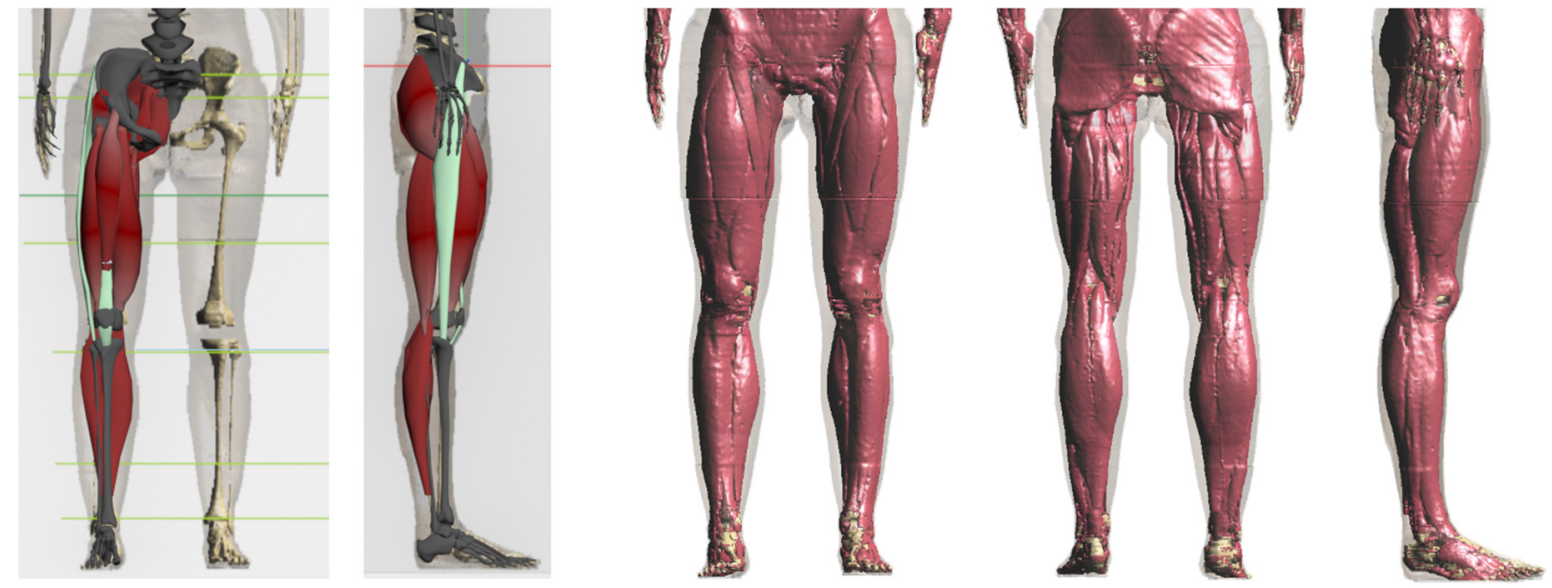

Fig. 13: Muscles of the legs modeled (left) and in MRI scans (right)

For the positioning and surface of the muscles the MRI data and anatomic are used. Highly important is the right position of the starting and end points of the muscles because they define the movement of the bones, the muscles and the skin.

After adding muscles in the thigh and calves, knee ligament and patella and considering the interaction between them, the muscles bulge and bend at movement. The ligament keeps its length and defines the endpoint of the muscle in the front of the thigh (see image 13, left and 14)
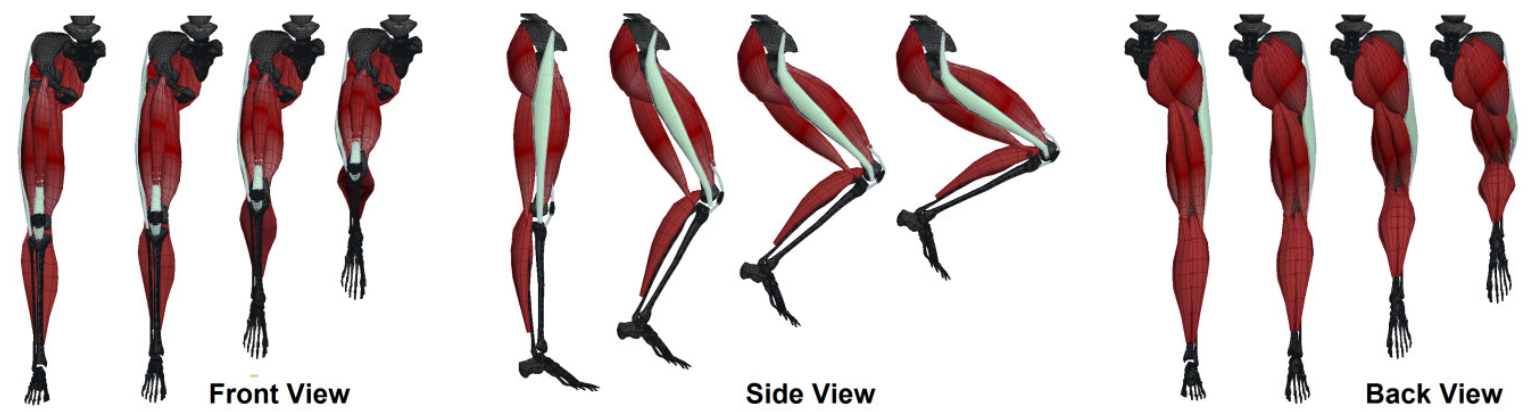

Fig. 14: Modeled muscles in movement (front, side, back view)

The system can be modified later by adding more muscles or tissues. Even already animated scan data can be supplemented by new anatomical structures. The muscles are modeled and animated in a rough way to test the skin deformations of each muscle in an early stadium. Later it is refined and the influence of each muscle is written into a deformation matrix. Depending on the combination and strength of the considerable muscles an overall deformation matrix is calculated and imported into the human model. 


\section{Adapting Template to Scan Data}

After defining all relevant muscles, the template model is adapted to the size and posture of the individual scan (see figure 15.a). To do this programmatically landmarks are used.
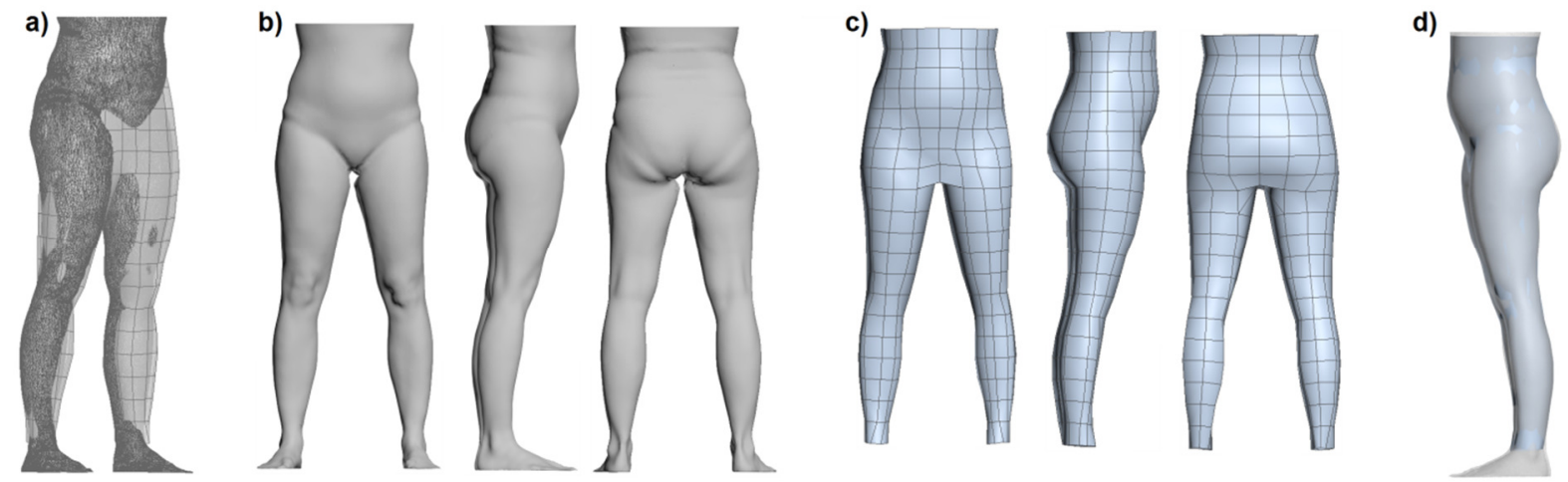

Fig. 15: Adapting the surface of the template model to the scan model: a) adapting the size and posture, b) the can model, c) the adapted surface, d) both models overlayed.

The surface of the scan data is assimilated programmatically by moving each vertex perpendicular to the corresponding bone. The process is identical to the process shown in figure 5 and 6 . the result is displayed in Fig. 15.c and d. The skin is refined now. As the area around the knee makes the biggest movement this faces are divided into $4 \times 4=16$ parts, the faces in the front of the thigh are divided into $3 \times 3=9$ parts. All other faces are doubled per side $=4$ faces. All new inserted vertices are moved slightly on the intersection planes to match the surface.
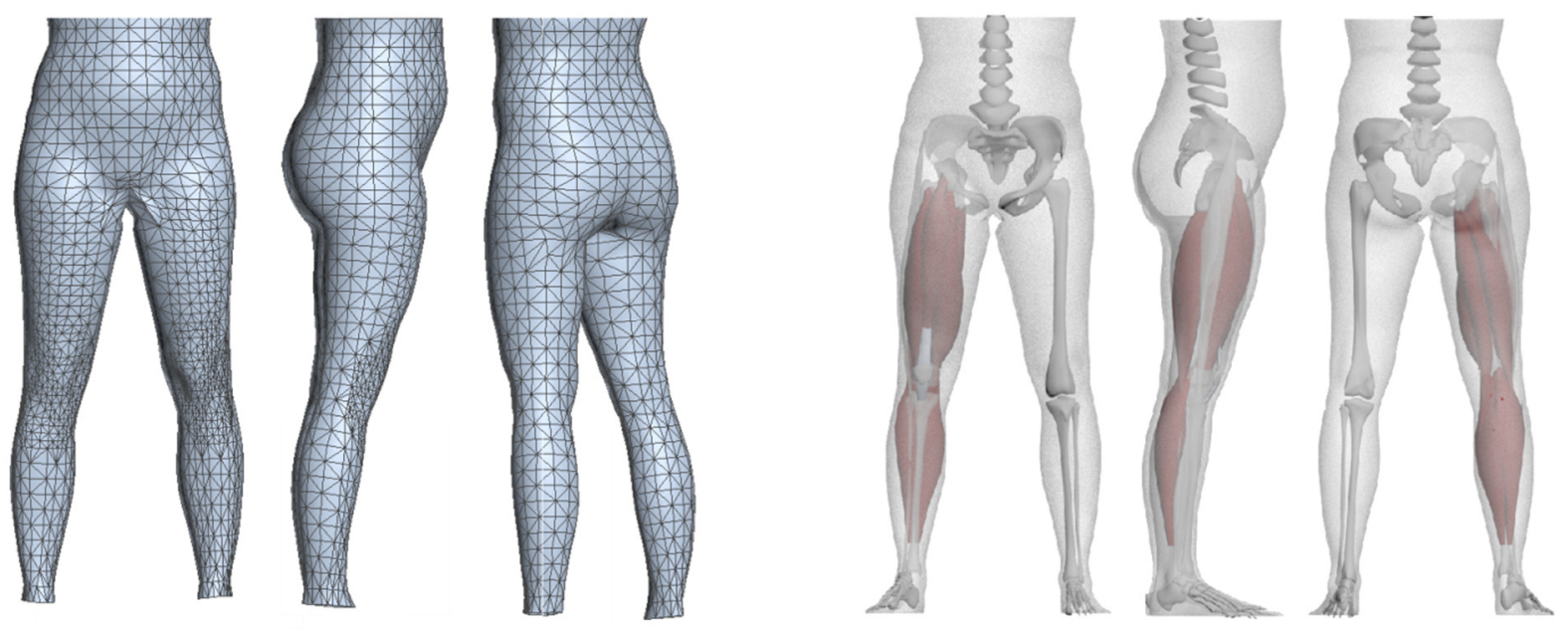

Fig. 16: Refined and Re-Adapted mesh (left) - Scaled and fitted skeleton and muscles to the scan model (right)

The effect of all postural muscles and tendons is transferred. The skeletal muscles can be imported in a defined and individual way depending on the characteristics of the muscular system of the scanned person.

The scan data is available now as a 3-dimensional skinned and rigged character with a triangular mesh surface, an anatomical skeleton and a muscular system. It is considering muscle work and can perform human movements by key framing. Postures and movement can be self-defined or taken from pre-defined or motion capturing data. They can be imported and reapplied at runtime to any scanned model that went through the afore-mentioned process. 


\section{Validation}

To validate the anatomical correct movement of the skin a scan model has been scanned in different postures. The developed model was adapted to the standing posture of this model and brought then into the scan postures. Fig. 17 shows two of that postures (top and bottom).
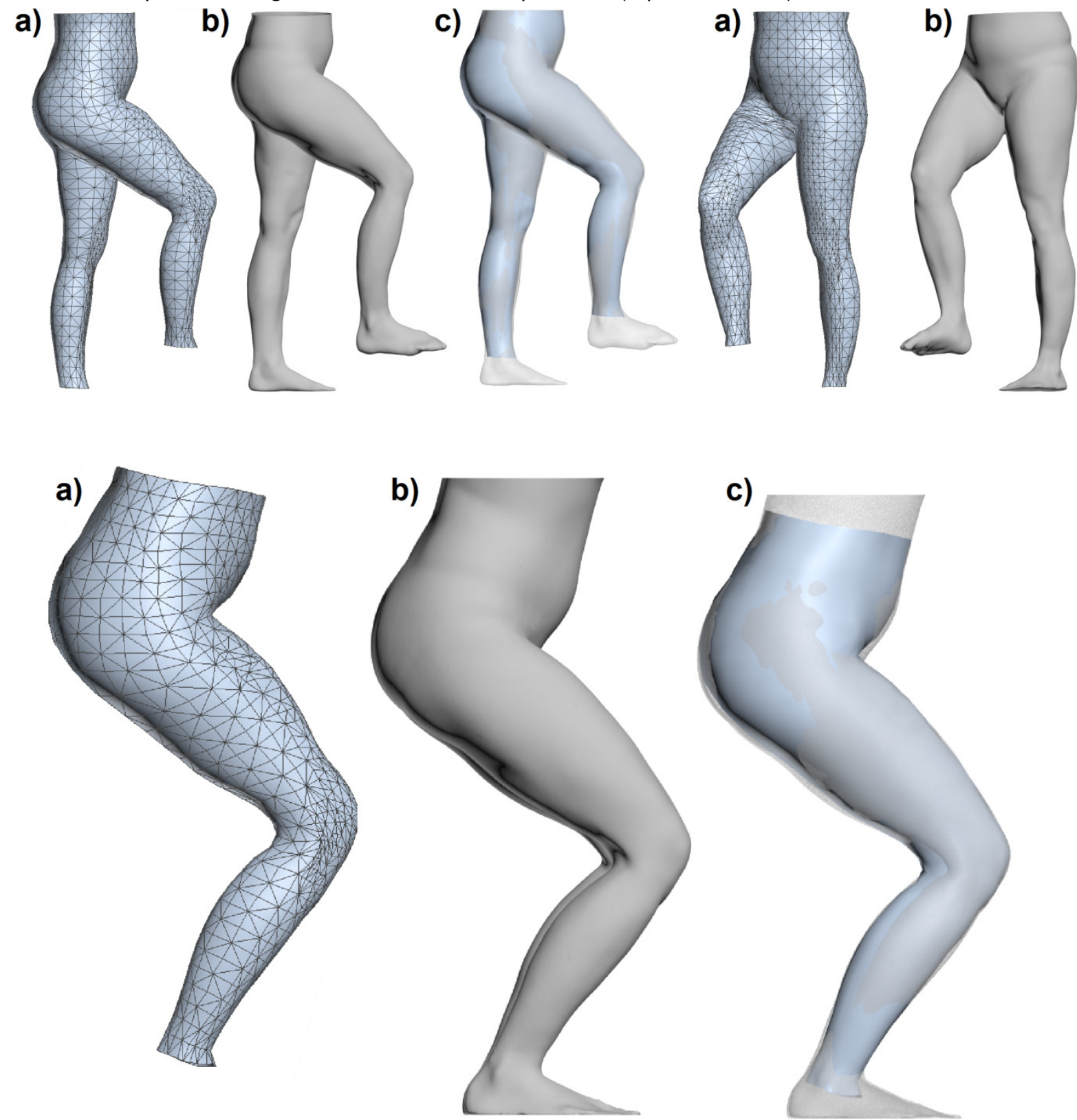

Fig. 17: Two sample scan postures: a) adapted template models, b) scan models, c) both models overlayed.

The quality of the results is quite good and sufficient for constructing clothes based on them. If a finer mesh is required for an use case, the faces can be divided as often as necessary and adapted to the surface, the weights of the new vertices can be calculated.

\section{Results}

We presented a highly automated methodology for transforming individual scan data into realistic animated human models by adapting the scan data to a template model based on anthropometric coherences. This approach is fast and useful for adopting scan models to different postures, e.g. of athlets or wheel-chaired people. These poses can be used for garment design. 


\section{References}

[1] Schünke, Michael and Schulte,Erik and Schumacher, Udo and Voll, Markus and Wesker, Karl "Prometheus, LernAtlas der Anatomie", Georg Thieme Verlag, 2007, 2. Auflage

[2] Brett, Allen "Learning Body Shape Models from Real-world Data", PhD at University of Washington, 2005

[3] Scheepers, Ferdi and Parent, Richard E. and Carlson, Wayne E. and May, Stephen F., "Anatomy-based Modeling of the Human Musculature" in Proceedings of the 24th Annual Conference on Computer Graphics and Interactive Techniques, Siggraph 1997 http://dx.doi.org/10.1145/258734.258827

[4] Anguelov, Dragomir "Learning models of shape from 3D range data", PhD at Stanford University, 2005

[5] Christ, Andreas and Kainz, Wolfgang and Hahn, Eckhart G. and Honegger, Katharina and Zefferer, Marcel and Neufeld, Esra and Rascher, Wolfgang and Janka, Rolf and Bautz, Werner and Chen, Ji and Kiefer, Berthold and Schmitt, Peter and Hollenbach, Hans-Peter and Shen, Jianxiang and Oberle, Michael and Szczerba, Dominik and Kam, Anthony and Guag, Joshua W. and Kuster, Niels "The Virtual Family-development of surface-based anatomical models of two adults and two children for dosimetric simulations" in Physics in Medicine and Biology, 2010, http://stacks.iop.org/0031-9155/55/i=2/a=N01

[6] Laurent Moccozet and Fabien Dellas and Nadia Magnenat-thalmann and Silvia Biasotti and Michela Mortara and Bianca Falcidieno and Patrick Min and Remco Veltkamp "Animatable Human Body Model Reconstruction from 3D Scan Data Using Templates" in Proceedings of Workshop on Modelling and Motion Capture Techniques for Virtual Environments, CAPTECH'2004

[7] Dicko Ali-Hamadi and Tiantian Liu and Benjamin Gilles and Ladislav Kavan and Francois Faure and Olivier Palombi and Marie-Paule Cani "Anatomy Transfer" in ACM Transactions on Graphics (proceedings of ACM SIGGRAPH ASIA), 2013

[8] Meixner, Christine and Krzywinski, Sybille " Automated generation of human models from scan data in different, anatomically correct postures for rapid development of close-fitting, functional garments" in Proceedings of the 2nd International Scientific Conference "Intelligent Textiles and Mass Customisation" ITMC 2009

[9] Kirchdörfer, Elfriede and Mahr-Erhardt, Angela and Morlock, Simone "3D Body Scanning Utilization of 3D Body Data for Garment and Footwear Design" in 2nd International Conference on 3D Body Scanning Technologies, Lugano, 2011

[10] Pastura, F.C.H. and de Almeida, G.L. and Cunha, C. "Joint angles calculation through augmented reality" in Proceedings of The 2nd International Digital Human Modeling Symposium 2013 